\title{
Gamma-induced Degradation Analysis of Commercial off-the-shelf Camera Sensors
}

\author{
Bilal Aslam ${ }^{1}$, Sangeet Saha ${ }^{1}$, Zeba Khanam ${ }^{1}$, Xiaojun Zhai ${ }^{1}$, Shoaib Ehsan ${ }^{1}$, Rustam Stolkin ${ }^{2}$ and Klaus McDonald-Maier ${ }^{1}$ \\ ${ }^{1}$ Embedded and Intelligent Systems Lab, University of Essex, Colchester CO4 3SQ \\ ${ }^{2}$ Extreme Robotics Lab (ERL), University of Birmingham, Birmingham B15 2TT
}

\begin{abstract}
In this work, we investigated the degradation of commercial camera sensors exposed to $100 \mathrm{~Gy}$ of $\gamma$ dose at dose rates of $0.55 \mathrm{~Gy} / \mathrm{min}$ and $1.34 \mathrm{~Gy} / \mathrm{min}$ respectively. The results show that the degradation is strongly dependent on the dose rate but doesnt vary much with the accumulation of dose at constant dose rate. Furthermore, cameras with in-built processing electronics are more susceptible to gamma radiations as compared to the cameras with sensing unit only.
\end{abstract}

\section{INTRODUCTION}

Since the inception of commercialization of nuclear energy, the access to nuclear sites have been constrained due to the risk associated with presence of radiations. Robotic system emerges as an ideal solution to encounter this problem by providing data on the state of such places while minimizing the human intervention. However, in order to operate autonomously, a robot needs to understand its environment for the navigation. In contrast to previously employed range sensors like acoustic sensors and LIDAR (Light Detection and Ranging), now a days robots deploy vision sensors ranging from RGB-D sensors to stereo-cameras. Nuclear environments usually have high presence of penetrative rays like $\gamma$ and particles like neutron. These rays and particles can damage the sensors and thus compromise the reliability of the robots visual sensing system [1]. Camera systems built with radiationhardened components can be more robust to radiations [2], [3], [4], however, the associated excessive cost restrict their frequent deployment. Recent advances in sensor and imaging technologies have led to employment of the commercial offthe-shelf (COTS) digital cameras for robots due to their ease of use, low cost, compact size, low weight, and compact data storage [5].

In continuation of above discussion, it can be said that mathematical models predicting the degradation of commercial camera sensors for different types and levels of radiations is a very relevant research area. Apart from handful of related research work [6], [7], [8], the efforts towards measuring the degradation effect of COTS cameras due to radiation is still in its infancy. This research work is an effort to contribute in this domain. Different commercial cameras have been tested in $\gamma$ radiation facility and performance comparison is made between cameras having distinct sensor and processing unit and the ones with complex processing electronics along with the sensor. Furthermore, an effort has been made to differentiate between the effects of the dose rate and absorbed dose on the camera sensor.

\section{EXPERIMENTAL REALIZATION}

\section{A. Experimental Setup}

1) Dalton Cumbrian Facility (DCF): $\gamma$ radiation experiments were conducted with the $C o-60$ self shielded irradiator at the DCF, Cumbria, UK [9]. This $\gamma$ radiation source can provide a maximum dose rate of $(680 \mathrm{~Gy} / \mathrm{min})$ [9]. Depending on the distance from the source to the DUT (Device Under Test), the absorbed dose can be varied from $400 \mathrm{~Gy} / \mathrm{min}$ to approximately $4 \mathrm{~Gy} / \mathrm{min}$ and can be reduced to 0.06 $\mathrm{Gy} / \mathrm{min}$ with attenuation. Thus, the facility is suitable for experimentation under a range of dose rates. The experimental setup employed in this research work is shown in Figure 1.

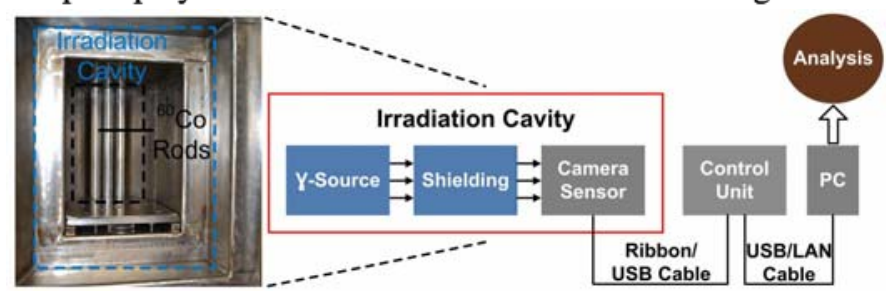

Fig. 1: Experimental Setup @DCF, UK

2) Test samples: The samples used in this experiment are low cost COTS camera sensors i.e., i) Raspberry Pi Camera [10], ii) Trust Usb Camera [11], iii) Spy Camera with wifi technology [12].

\section{B. Evaluation Metrics}

The standard procedure for complete characterization of the image sensors involves measuring the sensor output in both complete dark and under uniform light. In this research work, the dark response of the image sensor has been measured, and from that response, corresponding metrics have been evaluated. The following describes the considered metrics.

- Dark Current: Dark current is the leakage current that flows through the photosensitive devices under dark conditions (no photons are entering the device). The source of this current are the randomly generated electron-hole pairs owing to the defects in the silicon structure [13] which accelerates with increment in temperature. As per EMVA1288 [13] standard, the dark current is given as:

$$
\mu_{d}=\mu_{d, 0}+\mu_{I} t_{e x p}
$$




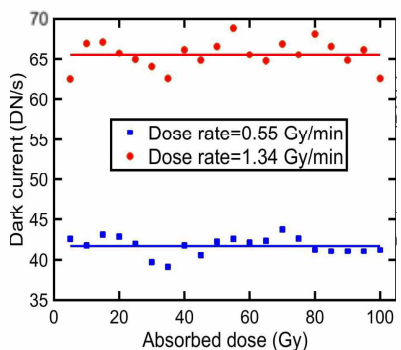

(a) Dark Current Vs Absorbed dose

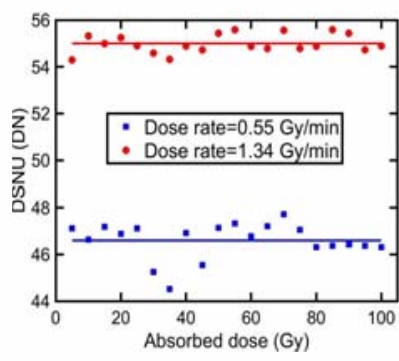

(b) DSNU Vs Absorbed dose

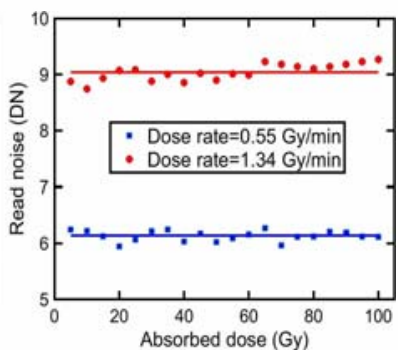

(c) Read Noise Vs Abosrbed dose

Fig. 2: Evaluated Metrics of Raspberry Pi camera as a function of absorbed dose

where $\mu_{d}$ is the average dark signal, $\mu_{d, 0}$ is the average dark signal for zero exposure time, $t_{\exp }$ is the exposure time, and $\mu_{I}$ is the mean dark current.

- Dark Signal Non-Uniformity (DSNU): DSNU is a form of fixed pattern noise (FPN) under no illumination. It is the spatial variation of the dark signal from pixel to pixel in the sensor array. The origin of this noise is the pixel mismatch across the photo detector area [13]. As per EMVA1288 standard, the DSNU is given as:

$$
\begin{gathered}
S_{y, \text { dark }}^{2}=\frac{1}{M N-1} \sum_{m=0}^{M-1} \sum_{n=0}^{N-1}\left(y_{\text {dark }}[m][n]-\mu_{y, \text { dark }}\right)^{2} \\
D S N U=S_{y, \text { dark }}
\end{gathered}
$$

where $M$ and $N$ are the number of rows and columns of the image, $m$ and $n$ are the row and column indices of the array, $y_{\text {dark }}[m][n]$ is the mean dark signal of all $[m][n]$ pixels in all the captured dark images, $\mu_{y, \text { dark }}$ is the mean gray value of all the captured dark images, and $S_{y, \text { dark }}^{2}$ is the spatial variance of all the dark images.

- Read Noise (RN): Read noise is essentially the noise level under no illumination which originates from the electronic circuitry of sensor. It is calculated by evaluating the temporal signal variance over a series of frames for individual pixel and then by taking the average over all the pixels, which identifies the noise over the image [13]

$$
\begin{gathered}
\sigma_{y, d a r k}^{2}=\frac{1}{K N} \sum_{j=1}^{K} \sum_{i=1}^{N}\left(P_{i j}-M\right)^{2} \\
R N=\sigma_{y, d a r k}
\end{gathered}
$$

where $K$ and $N$ represent the total number of acquired frames total number of pixels per frame, respectively, $P_{i j}$ is the $j^{\text {th }}$ pixel value from $i^{\text {th }}$ frame and $M$ is the mean value of all the $j^{\text {th }}$ pixels of $N$ frames.

\section{Evaluation Methodology}

1) Capture a set of $\eta$ number of dark frames* at different exposure times (varying from $10 \mathrm{~ms}$ to $1300 \mathrm{~ms}$ ) and calculate the mean dark frame at each exposure time.

${ }^{*}$ In our experimental setup, $\eta$ is considered as 5
2) Calculate the dark current by computing the mean response of $\frac{\left(I m g_{\zeta^{\prime}}-I m g_{\zeta^{\prime}}\right)}{\left(\delta t_{\exp }\right)} . I m g_{\zeta}$ and $I m g_{\zeta^{\prime}}$ are the images captured at $10 \mathrm{~ms}$ and $1300 \mathrm{~ms}$ of exposure time, respectively, and $t_{\exp }$ is the difference in the exposure times, i.e. $1290 \mathrm{~ms}$. DSNU is measured as the standard deviation of the response of difference image $\left(I m g_{\zeta}-I m g_{\zeta^{\prime}}\right)$.

3) Calculate the read noise by using equation 5. Utilize the images captured at small integration time $(10 \mathrm{~ms})$ to minimize the influence of DSNU.

We have carried out the above three steps for each distinct dose rate. In our experiment, the dose rates were $0.55 \mathrm{~Gy} / \mathrm{min}$ and $1.34 \mathrm{~Gy} / \mathrm{min}$, respectively.

\section{RESUltS AND ANALYSIS}

TABLE I: Evaluated Metrics under $\gamma$ dose

\begin{tabular}{|c|c|c|c|}
\hline Metric & $\sigma_{1}(\%)^{*}$ & $\sigma_{2}(\%)^{\dagger}$ & $\Delta(\%)^{\ddagger}$ \\
\hline Dark Current & \pm 7.1 & \pm 5.3 & 57.1 \\
\hline DSNU & \pm 5.2 & \pm 1.5 & 18.03 \\
\hline Read Noise & \pm 1.6 & \pm 2.8 & 48.4 \\
\hline
\end{tabular}

Figure 2 depicts the metrics of interest plotted as function of absorbed dose for two different dose rates. All the metrics exhibit significant degradation under the gamma dose. There are two important observations that are evident from the depicted graphs. The first observation is that for the same absorbed dose, the higher dose rate causes more degradation. The second observation is that the metrics degradation does not vary significantly with the accumulation of the dose. Therefore, it is the initial dose and not the cumulative dose that is more detrimental to the measured metrics. These observations are detailed in the Table 1.

Figure 3a shows the histogram of images captured from two Raspberry Pi cameras (with same exposure time) radiated to same dose but at different dose rates. The dark image from the camera radiated at $1.34 \mathrm{~Gy} / \mathrm{min}$ has ten times more hot

${ }^{*} \sigma_{1}=$ Maximum swing of the metric value around the mean value as absorbed dose is varied from $5 \mathrm{~Gy}$ to $100 \mathrm{~Gy} @ 0.55 \mathrm{~Gy} / \mathrm{min}$

${ }^{\dagger} \sigma_{2}=$ Maximum swing of the metric value from the mean value as absorbed dose is varied from 5 Gy to $100 \mathrm{~Gy} @ 1.34 \mathrm{~Gy} / \mathrm{min}$;

$\ddagger \Delta$ = Increase in the metric value as dose rate is varied from $0.55 \mathrm{~Gy} / \mathrm{min}$ to $1.34 \mathrm{~Gy} / \mathrm{min}$ 


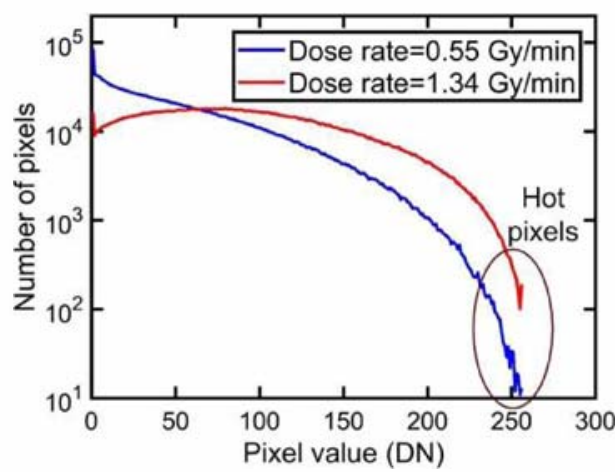

(a) Dark image histogram as a function of dose rate

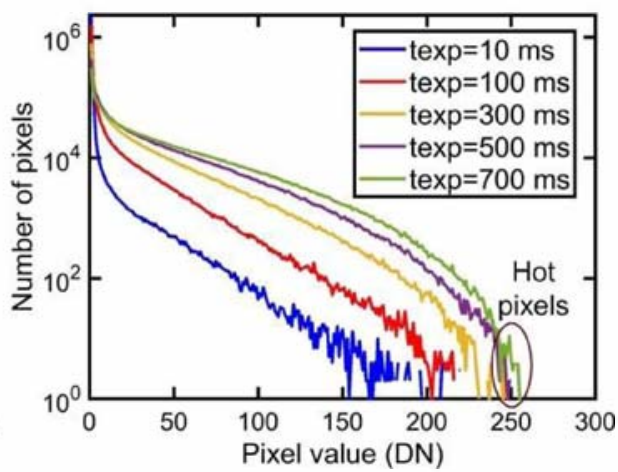

(b) Dark image histogram @100 Gy for differenet exposure time

Fig. 3: Representation of dark image histogram

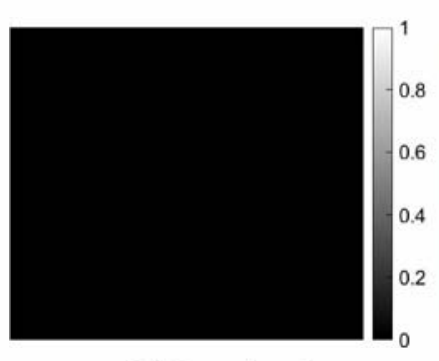

(a) Pre-radiated

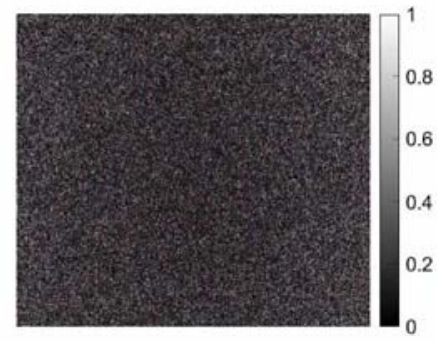

(b) $100 \mathrm{~Gy} @ 0.55 \mathrm{~Gy} / \mathrm{min}$

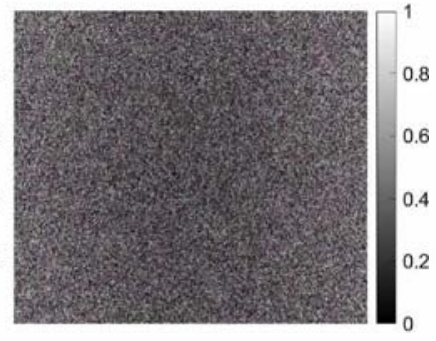

(c) $100 \mathrm{~Gy} @ 1.34 \mathrm{~Gy} / \mathrm{min}$

Fig. 4: Dark images obtained during $\gamma$ radiation

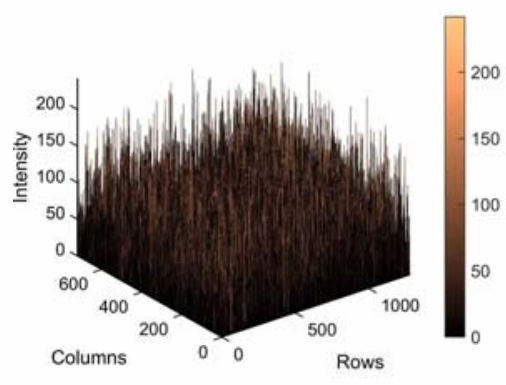

(a) Raspberry Pi camera

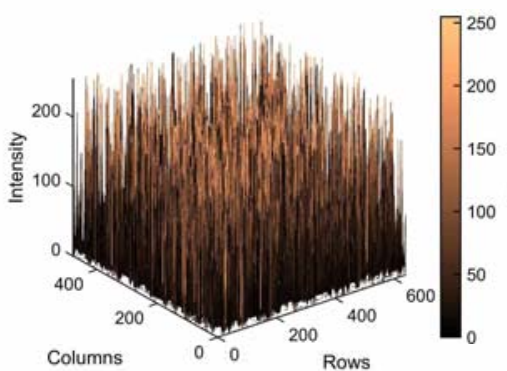

(b) Trust usb camera

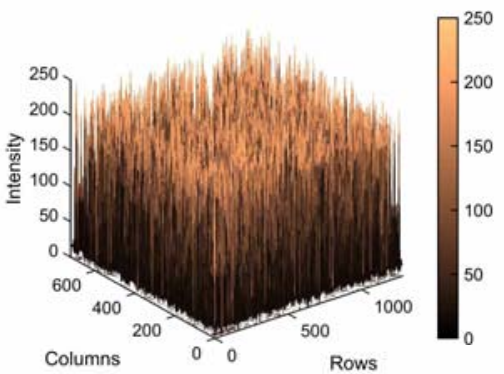

(c) Spy camera with Wifi

Fig. 5: 3D surface plot of images captured from radiated cameras @ $100 \mathrm{~ms}$ of exposure time

pixels compared to the one radiated at $0.55 \mathrm{~Gy} / \mathrm{min}$. Figure $3 \mathrm{~b}$ shows the histogram plot of the images captured with different exposure times from a radiated camera sensor. The presence of hot pixels at higher exposure times is evident. Figure 4 displays the dark images captured from the Raspberry Pi camera at same exposure time before and after the radiation. The image captured from the camera radiated at higher dose rate appears significantly brighter, thus depicting more degradation. Finally, Figure 5 provides a comparison of the gamma-induced degradation in camera with only a sensor unit (Raspberry Pi camera) to the more complex cameras (Trust and Spy) with combined sensing and processing units. The complex cameras have a much higher dark signal at the same exposure time under identical gamma dose, owing to the additional noise of the processing electronics. The Spy camera with much smaller pixel size as compared to the Trust camera, develop a much higher dark signal. Furthermore, it has been observed that the wireless connectivity with the Spy camera remains intact after absorbing dose of $100 \mathrm{~Gy}$.

\section{CONCLUSION}

Experiments were performed to evaluate the $\gamma$-induced performance degradation of COTS camera sensors. The evaluation metrics included dark current, DSNU and read noise. In future experiments, the metrics related to the uniformly illuminated camera sensor under the $\gamma$ dose will be evaluated to completely characterize the sensor in the radiation environment. 


\section{ACKNOWLEDGEMENT}

This work is supported by the UK Engineering and Physical Sciences Research Council through grants EP/R02572X/1 and EP/P017487/1.

\section{REFERENCES}

[1] K. Nagatani, S. Kiribayashi, Y. Okada, K. Otake, K. Yoshida, S. Tadokoro, T. Nishimura, T. Yoshida, E. Koyanagi, M. Fukushima et al., "Gamma-ray irradiation test of electric components of rescue mobile robot quince," in 2011 IEEE International Symposium on Safety, Security, and Rescue Robotics. IEEE, 2011, pp. 56-60.

[2] S. Rizzolo, V. Goiffon, F. Corbière, R. Molina, A. Chabane, S. Girard, P. Paillet, P. Magnan, A. Boukenter, T. Allanche et al., "Radiation hardness comparison of cmos image sensor technologies at high total ionizing dose levels," IEEE Transactions on Nuclear Science, vol. 66, no. 1, pp. 111-119, 2019.

[3] V. e. a. Goiffon, "Multi-mgy radiation hard cmos image sensor: Design, characterization and x/gamma rays total ionizing dose tests," IEEE Transactions on Nuclear Science, vol. 62, no. 6, pp. 2956-2964, 2015.

[4] —, "Total ionizing dose effects on a radiation-hardened cmos image sensor demonstrator for iter remote handling," IEEE Transactions on Nuclear Science, vol. 65, no. 1, pp. 101-110, 2018.

[5] Z. J. Diggins, N. Mahadevan, D. Herbison, G. Karsai, E. Barth, R. A. Reed, R. D. Schrimpf, R. A. Weller, M. L. Alles, and A. Witulski, "Range-finding sensor degradation in gamma radiation environments," IEEE Sensors Journal, vol. 15, no. 3, pp. 1864-1871, 2014.

[6] Z. Wang, S. Huang, M. Liu, Z. Xiao, B. He, Z. Yao, and J. Sheng, "Displacement damage effects on cmos aps image sensors induced by neutron irradiation from a nuclear reactor," AIP advances, vol. 4, no. 7, p. 077108, 2014.

[7] M. Dayton, P. Datte, A. Carpenter, M. Eckart, A. Manuel, H. Khater, D. Hargrove, and P. Bell, "Radiation effects on active camera electronics in the target chamber at the national ignition facility," in Target Diagnostics Physics and Engineering for Inertial Confinement Fusion VI, vol. 10390. International Society for Optics and Photonics, 2017, p. 1039007.

[8] C. Virmontois, A. Toulemont, G. Rolland, A. Materne, V. Lalucaa, V. Goiffon, C. Codreanu, C. Durnez, and A. Bardoux, "Radiationinduced dose and single event effects in digital cmos image sensors," IEEE Transactions on Nuclear Science, vol. 61, no. 6, pp. 3331-3340, 2014.

[9] "Dalton cumbrian facility" http://www.dalton.manchester.ac.uk/ recycle-bin/daltoncumbrianfacility/discover/facilities/co-60irradiator/.

[10] "Raspberry pi camera," https://www.raspberrypi.org/products/ camera-module-v2/.

[11] "Trust usb camera," https://www.trust.com/en/trust/webcam.

[12] "Spy camera," https://www.eyetek.co.uk/product-category/ wireless-spy-cameras/.

[13] E. M. V. Association et al., "Emva standard 1288, standard for characterization of image sensors and cameras," Release, vol. 3, p. 29, 2010. 